
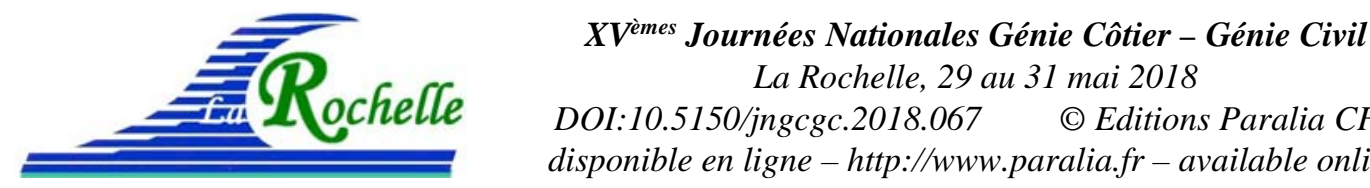

DOI:10.5150/jngcgc.2018.067 ～(C) Editions Paralia CFL

disponible en ligne - http://www.paralia.fr - available online

\title{
Analyse de données de vent en Europe pour caractériser le potentiel éolien en mer
}

\section{Léa THIEBAUD ${ }^{1}$, Anne-Laure TIBERI-WADIER ${ }^{1}$, Yvonnick BEAUDOUIN ${ }^{2}$}

1. Cerema Eau mer et fleuves, 155 rue Pierre Bouguer, 29280 Plouzané, France. lea.thiebaud@cerema.fr ; anne-laure.tiberi-wadier@cerema.fr

2. Institut Universitaire Européen de la Mer, rue Dumont d'Urville, 29280 Plouzané, France.yvonnick.beaudouin@univ-brest.fr

\section{Résumé :}

Dans le cadre de travaux sur la connaissance du potentiel éolien en mer, le Commissariat Général au Développement Durable du Ministère de la Transition Ecologique et Solidaire a demandé au Cerema d'effectuer une analyse de données de vent existantes en mer, au large de pays européens développant ces technologies. Des données issues de modèles météorologiques et de mesures in situ ont ainsi été collectées puis analysées pour :

- identifier les écarts entre modèles et mesures ;

- caractériser des variations spatiales et temporelles du vent ;

- identifier et comparer des formules pour l'extrapolation verticale du vent ;

- déterminer un facteur de charge théorique pour les éoliennes en mer.

Les jeux de données étudiés sont globalement cohérents. Les écarts les plus importants se situent à proximité des côtes et diminuent au large, là où les vitesses augmentent.

Les moyennes mensuelles et horaires du vent ont été représentées sur des cycles annuels et journaliers de manière à identifier les périodes de vents forts et faibles bien que le régime de vent présente également des variations interannuelles.

Plusieurs formules tenant compte des caractéristiques de la surface de la mer ont été testées pour extrapoler verticalement la vitesse du vent. Les vitesses de vent du modèle CFSR à 10 mètres ont été estimées aux hauteurs balayées par les pâles des éoliennes. Une courbe de production éolienne théorique a ensuite été utilisée pour calculer le facteur de charge sur la zone d'étude.

Les résultats obtenus pourraient être améliorés en utilisant des données entrantes plus précises et en ayant une meilleure connaissance des courbes de production des technologies éoliennes actuelles.

Mots-clés : Vent, Eolien, Europe, Mer, Modèle, Mesure, Ré analyse, Variation, Vitesse, Facteur de charge, Production, Ecarts.

\section{Introduction}

Les vitesses importantes du vent et la faible profondeur des mers d'Europe du Nord rendent ces secteurs favorables pour l'implantation de parcs éoliens en mer posés. Le 


\section{Thème 5 - Énergies et ressources marines}

Royaume-Uni, le Danemark, l'Allemagne, la Belgique et les Pays-Bas sont les pays les plus avancés dans ce domaine. À ce jour, la France n'a installé aucune éolienne en mer mais plusieurs projets de parcs commerciaux éoliens posés sont en cours en Manche et en Atlantique. Des fermes pilotes éoliennes flottantes sont également prévues en Atlantique et en mer Méditerranée afin de préparer le déploiement commercial de cette filière. Une connaissance précise de la ressource en vent sur plusieurs années et à l'échelle de différents pays peut permettre de mieux caractériser le potentiel de l'éolien en mer afin de planifier le développement de cette technologie.

\section{Méthodologie}

\subsection{Recueil des données}

Les données étudiées proviennent de ré analyses météorologiques et de mesures in situ réalisées par des anémomètres fixés sur des mâts. La zone d'étude correspond à la partie nord-ouest de l'Europe. Les fournisseurs de données sont :

- l'Agence américaine d'observation océanique et atmosphérique (National Oceanic and Atmospheric Administration NOAA) qui met à disposition les données climatologiques du modèle CFSR (Climate Forecast System Reanalysis) ;

- le Centre européen pour les prévisions météorologiques à moyen terme (European Centre for Medium-Range Weather Forecasts ECMWF) qui met à disposition les données climatologiques du modèle Era Interim ;

- le gouvernement Allemand qui met à disposition les données des plateformes de recherche FINO ;

- le Crown Estate qui met à disposition des données maritimes via le site Marine Data Exchange.

Tableau 1. Synthèse des données étudiées.

\begin{tabular}{|c|c|c|c|c|}
\hline Données & Type & Résolutions & Paramètres & Hauteurs (m) \\
\hline $\begin{array}{l}\text { CFSR } \\
(N O A A)\end{array}$ & $\begin{array}{l}\text { Modèle } \\
1979-2010\end{array}$ & $\begin{array}{l}0,312^{\circ} * 0,312^{\circ} \\
1 \mathrm{~h}\end{array}$ & $\begin{array}{l}\text { Vent zonal, méridien, } \\
\text { cisaillement, rugosité }\end{array}$ & 10 \\
\hline $\begin{array}{l}\text { Era Interim } \\
(\text { ECMWF) }\end{array}$ & $\begin{array}{l}\text { Modèle } \\
1979-2010\end{array}$ & $\begin{array}{l}0,75^{\circ} * 0,75^{\circ} \\
6 h\end{array}$ & Vent zonal, méridien & 10 \\
\hline $\begin{array}{l}\text { FINO } 2 \\
\text { (Allemagne) }\end{array}$ & $\begin{array}{l}\text { Mesures } \\
2007-2017\end{array}$ & $\begin{array}{l}\text { Ponctuel } \\
10 \text { minutes }\end{array}$ & Vitesse, direction & $\begin{array}{l}32,42,52,62, \\
72,82,92,102\end{array}$ \\
\hline $\begin{array}{l}\text { Greater Gabbard } \\
\text { (Royaume-Uni) }\end{array}$ & $\begin{array}{l}\text { Mesures } \\
2005-2010\end{array}$ & $\begin{array}{l}\text { Ponctuel } \\
10 \text { minutes }\end{array}$ & Vitesse, direction & $\begin{array}{l}42,52,62,72, \\
82\end{array}$ \\
\hline
\end{tabular}




\section{XVèmes Journées Nationales Génie Côtier - Génie Civil \\ La Rochelle, 29 au 31 mai 2018}

Météo France produit également des données de vent en mer, d'une résolution spatiale fine de $0,025^{\circ}$, sur la période 2000 à 2015 à l'aide du modèle à maille fine Arome. Cependant ces données ne sont pas en accès libre et ne recouvrent qu'une partie de la zone d'étude. Elles n'ont donc pas été exploitées dans le cadre de ces travaux.

\subsection{Comparaisons des données}

Les vitesses de vent modélisées ou mesurées sont comparées entre elles à l'aide des équations (1) et (2).

Ecart entre moyennes des vitesses $=\overline{\text { vitesse }_{1}}-\overline{\text { vitesse }_{2}}$

Moyenne des valeurs absolues des écarts entre vitesses $=\overline{\mid \text { vitesse }_{1}-\text { vitesse }_{2} \mid}$

où vitesse 1 et vitesse 2 sont les séries de données de vent comparées en ne tenant compte que des dates communes aux deux séries.|

\subsection{Analyses des variations du vent}

Les variations du vent peuvent être étudiées sur différentes échelles spatiales et temporelles. Des mesures de vent avec une fréquence de $1 \mathrm{~Hz}$ pendant 10 minutes permettent de caractériser des phénomènes de rafales. Des données horaires sur plusieurs dizaines d'années permettent d'identifier des variations journalières et annuelles. Par ailleurs, les caractéristiques du vent dépendent de la rugosité du site. En mer, le vent est plus constant qu'à terre. Les interactions entre l'océan et l'atmosphère sont cependant complexes. Afin de mieux connaître le régime global du vent en mer sur la zone d'étude, les ré analyses CFSR de 1979 à 2010 ont été moyennées par heure et par mois de manière à représenter les variations quotidiennes et annuelles du vent.

\subsection{Extrapolation verticale du vent}

La vitesse du vent peut être extrapolée verticalement par une loi logarithmique (MOLIN, 2002).

$u(\mathrm{z})=u_{10} *\left(\frac{\ln \left(\frac{\mathrm{z}}{\mathrm{z}_{0}}\right)}{\ln \left(\frac{10}{\mathrm{z}_{0}}\right)}\right)$

où $u$ est la vitesse du vent, $u_{10}$ est la vitesse du vent à 10 mètres, $z$ l'altitude et $z_{0}$ la rugosité du site définie par la relation de Charnock en équation (4).

$z_{0}=\frac{\alpha_{c}{ }^{*} u *^{2}}{g}$

où $\alpha_{c}$ est la constante de Charnock et vaut $0,014, u_{*}$ est la vitesse de frottement et $g$ l'accélération de la pesanteur et vaut $9,81 \mathrm{~m} \cdot \mathrm{s}^{-2}$.

La vitesse de frottement et la rugosité du site ont été évaluées par trois méthodes.

a) Méthode 1

La vitesse de frottement est déterminée en calculant le coefficient de frottement qui dépend la vitesse du vent et de l'âge des vagues (MOLIN, 2002).

$u_{*}{ }^{2}=C_{d} * u_{10}$ 


\section{Thème 5 - Énergies et ressources marines}

$C_{d}=\left(1+6^{*} 10^{-2}\right) * 10^{-3} * u_{10}$

où $u_{*}$ est la vitesse de frottement, $u_{10}$ la vitesse du vent à 10 mètres et $C_{d}$ le coefficient de frottement.

b) Méthode 2

La vitesse de friction est déterminée à l'aide du cisaillement disponible sur le site de la NOAA pour chaque pas de temps horaire du modèle CFSR.

$u_{*}=\sqrt{\frac{|\tau|}{\rho}}$

où $\tau$ est la contrainte de cisaillement et $\rho$ est la masse volumique de l'air et vaut $1,225 \mathrm{~kg} \cdot \mathrm{m}^{-3}$.

c) Méthode 3

La rugosité zo du modèle CFSR est disponible sur le site de la NOAA pour chaque heure.

\subsection{Estimation du facteur de charge}

Un facteur de charge théorique est évalué à partir des vitesses extrapolées aux hauteurs balayées par les pâles de l'éolienne et d'une courbe de production éolienne.

La vitesse moyenne du vent sur le disque balayé par les pâles est définie par l'équation

(8) (SUMNER, 2006).

$\overline{\boldsymbol{u}}_{\text {disque }}=\frac{1}{A} * \iint_{\text {disque }} \boldsymbol{u}(\mathbf{z}) \boldsymbol{d A}$

où $\bar{u}_{\text {disque }}$ est la vitesse moyenne du vent sur le disque, $A$ l'aire du disque balayé par les pâles de l'éolienne, $u$ la vitesse du vent et $z$ l'altitude.

En pratique, le calcul de cette vitesse a été réalisé par une moyenne pondérée selon la surface des vitesses de vent pour chaque hauteur du disque découpé en tranche de $1 \mathrm{~m}$. La courbe de production est déterminée pour une éolienne en mer fictive de $8 \mathrm{MW}$ dont les caractéristiques sont proches des éoliennes actuelles. Le rotor est situé à 100 mètres de haut et les pâles mesurent $81,5 \mathrm{~m}$.
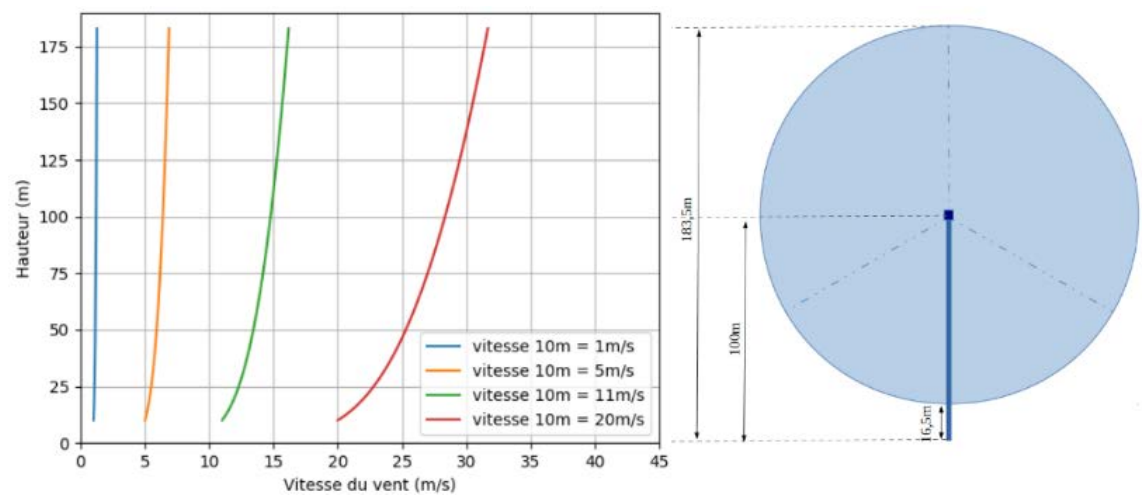

Figure 1. Profil vertical du vent sur la hauteur balayée par les pâles de l'éolienne. 


\section{XVèmes Journées Nationales Génie Côtier - Génie Civil \\ La Rochelle, 29 au 31 mai 2018}

L'éolienne démarre pour des vitesses de vents supérieures à $3 \mathrm{~m} / \mathrm{s}$ et s'arrête pour des vents supérieurs à $25 \mathrm{~m} / \mathrm{s}$. Pour des vents compris entre $3 \mathrm{~m} / \mathrm{s}$ et la vitesse nominale de l'éolienne la puissance est définie par l'équation (9).

Puissance $=\frac{1}{2} * C_{p} * \rho^{*} S^{*} \bar{u}_{\text {disque }}{ }^{3}$

où $\rho$ est la masse volumique de l'air et vaut $1,225 \mathrm{~kg} \cdot \mathrm{m}^{-3}, S$ la surface balayée par les pâles de l'éolienne, $\bar{u}_{\text {disque }}$ la vitesse du vent moyennée sur le disque balayé par les pâles de l'éolienne et $C_{p}$ est le coefficient de puissance (SUMNER, 2006 ; HENCKES et al., 2018 ; JOURDIER, 2015).

Le coefficient de puissance a été fixé à 0,4 mais il dépend des caractéristiques intrinsèques de l'éolienne. Il varie avec la vitesse de rotation de l'éolienne et la vitesse du vent.

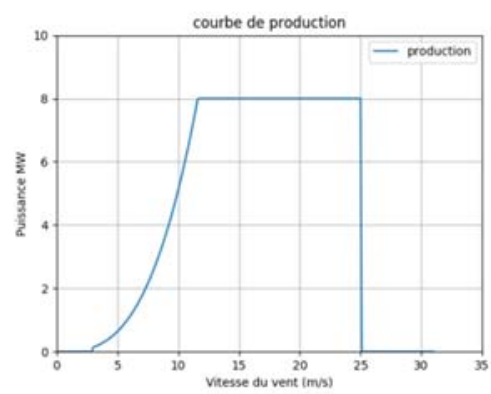

Figure 2. Courbe de production théorique pour une éolienne de $8 \mathrm{MW}$ avec un coefficient de puissance fixe à 0,4.

\section{Résultats}

3.1 Comparaison des ré analyses des modèles CFSR et Era Interim

Les ré analyses du modèle CFSR sont comparées à celles du modèle Era Interim. Les cartes de la figure 2 indiquent l'écart entre les moyennes des vitesses du vent et la moyenne des valeurs absolues des écarts entre les vitesses de vent de 1979 à 2010 . 


\section{Thème 5 - Énergies et ressources marines}
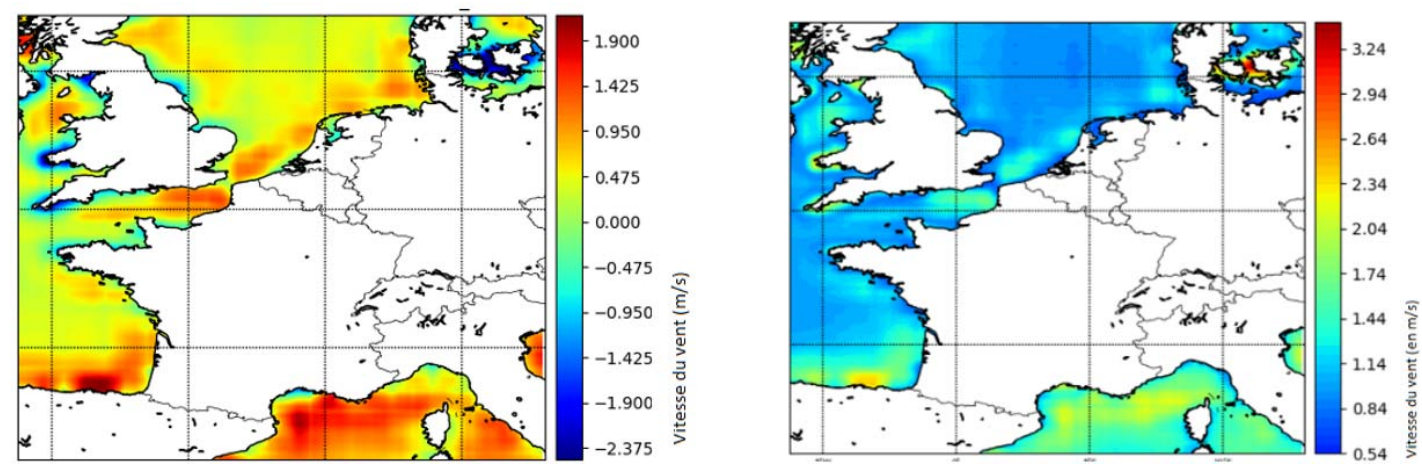

Figure 2. Comparaison des vitesses de vent à 10 mètres des modèles CFSR et Era Interim : écart entre les moyennes des vitesses (à gauche) et moyenne des valeurs absolues des écarts entre les vitesses (à droite) de 1979 à 2010.

Près des côtes du Royaume-Uni et en mer Baltique, la vitesse moyenne du vent du modèle CFSR est inférieure à celle du modèle Era Interim. En mer Méditerranée, en Mer du Nord, en Manche et en Atlantique les vitesses moyennes des ré analyses CFSR sont supérieures à celles du modèle Era Interim jusqu'à environ $40 \mathrm{~km}$ des côtes. Plus au large les écarts entre ces données diminuent. La moyenne des valeurs absolues des écarts entre les vitesses est maximale en mer Baltique $(3,3 \mathrm{~m} / \mathrm{s})$. Elle atteint $2 \mathrm{~m} / \mathrm{s}$ en Atlantique au large de l'Espagne et en Méditerranée.

\subsection{Analyses des variations spatiales et temporelles du vent à 10 mètres}

Les données CFSR ont été moyennées sur des cycles annuels et journaliers. Les moyennes des vitesses de vent pour les mois de janvier et juillet sont représentées pour :

- 31 années, de 1979 à 2010 ;

- les années qui présentent des moyennes mensuelles de vent faibles ;

- les années qui présentent des moyennes mensuelles de vent élevées.

Les moyennes de vent sont globalement plus élevées en hiver qu'en été. Il peut arriver cependant que les moyennes hivernales soient proches des moyennes estivales comme c'est le cas pour les mois de janvier 1997 et juillet 1998 (voir figure 3). Par ailleurs, on constate que pour des périodes de vents faibles les vitesses sont relativement homogènes sur la zone d'étude. Pendant ces périodes la production électrique d'origine éolienne en mer sera quasiment nulle pour tous ces pays.

Les variations du vent ont ensuite été étudiées sur un cycle journalier en six points de la zone d'étude. 


\section{XVèmes Journées Nationales Génie Côtier - Génie Civil La Rochelle, 29 au 31 mai 2018}

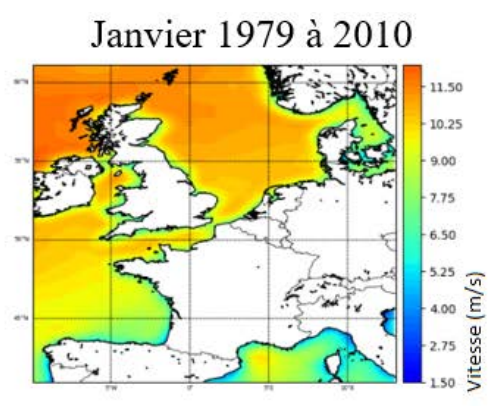

Juillet 1979 à 2010
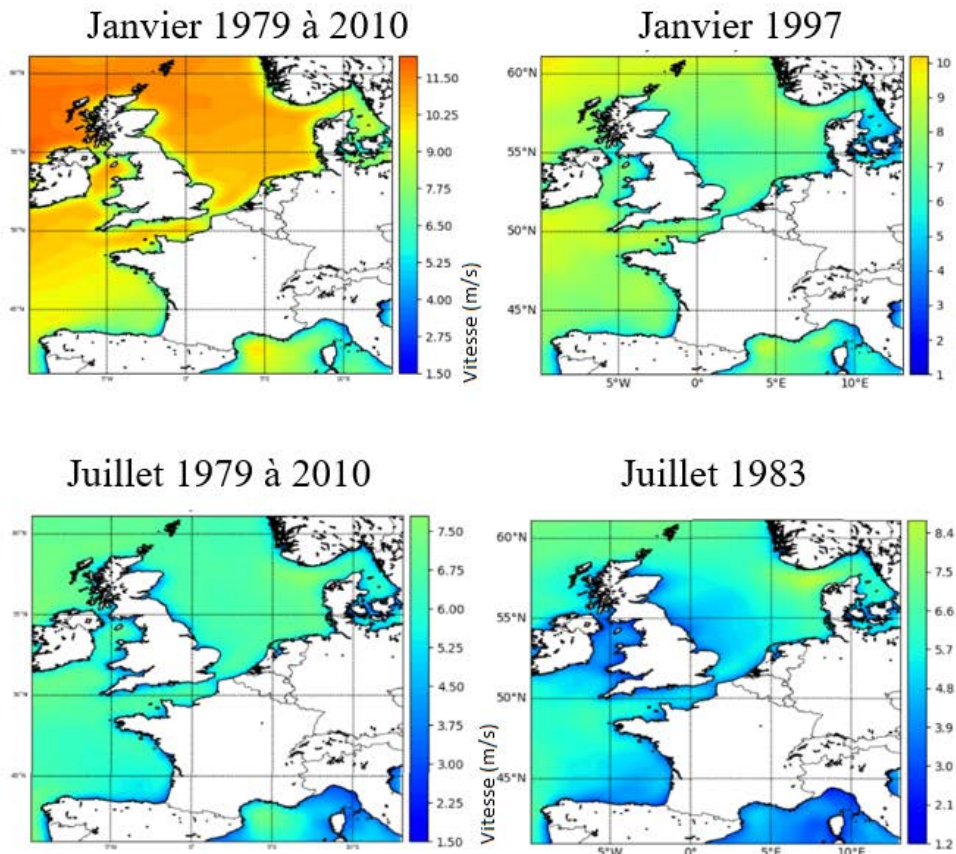

Juillet 1983

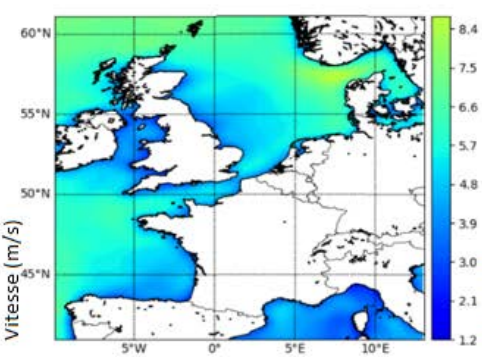

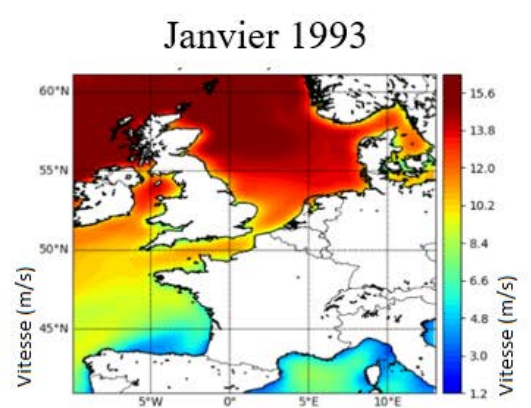

Juillet 1998

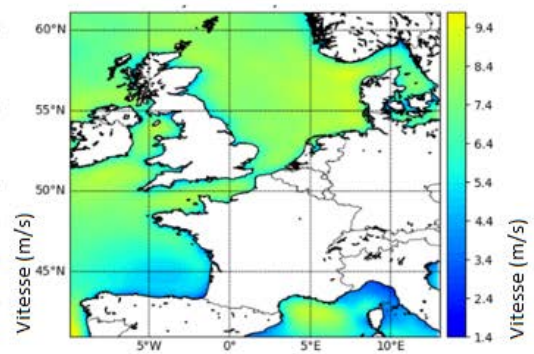

Figure 3. Moyennes mensuelles du vent pour les mois de janvier de 1979 à 2010 (en haut à gauche) et de mois de juillet de 1979 à 2010 (en bas à gauche), de janvier 1997

(en haut au milieu), de janvier 1993 (en haut à droite), de juillet 1983 (en bas au milieu) et de juillet 1998 (en bas à droite).
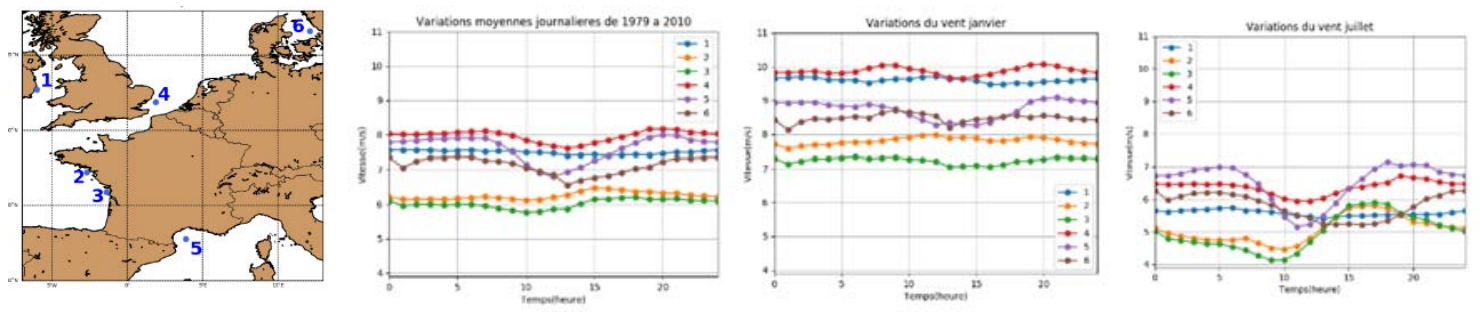

Figure 4. Variations des moyennes horaires du vent sur un cycle de 24 heures pour la totalité de la période 1979 - 2010 et pour les mois de janvier et de juillet sur cette même période.

Les données moyennées par heure permettent de visualiser des variations journalières plus marquées en été qu'en hiver notamment pour les points 2,3 et 5 situés au sud de la zone d'étude. En période estivale les phénomènes de brises de mer, qui se traduisent par une augmentation de la vitesse du vent en journée, peuvent être plus marqués en ces points. Par ailleurs, les vents d'ouest dominants en Europe aux latitudes de la zone d'étude (LAUGEL, 2013) peuvent expliquer le décalage temporel des extremums du vent. 


\section{Thème 5 - Énergies et ressources marines}

\subsection{Comparaison des méthodes d'extrapolation verticale du vent}

La moyenne des valeurs absolues des écarts (voir équation (2)) entre les ré analyses extrapolées verticalement aux hauteurs étudiées et les mesures in situ est calculée pour chaque altitude de mesure du vent. Les variations de ces écarts sont présentées figure 5.
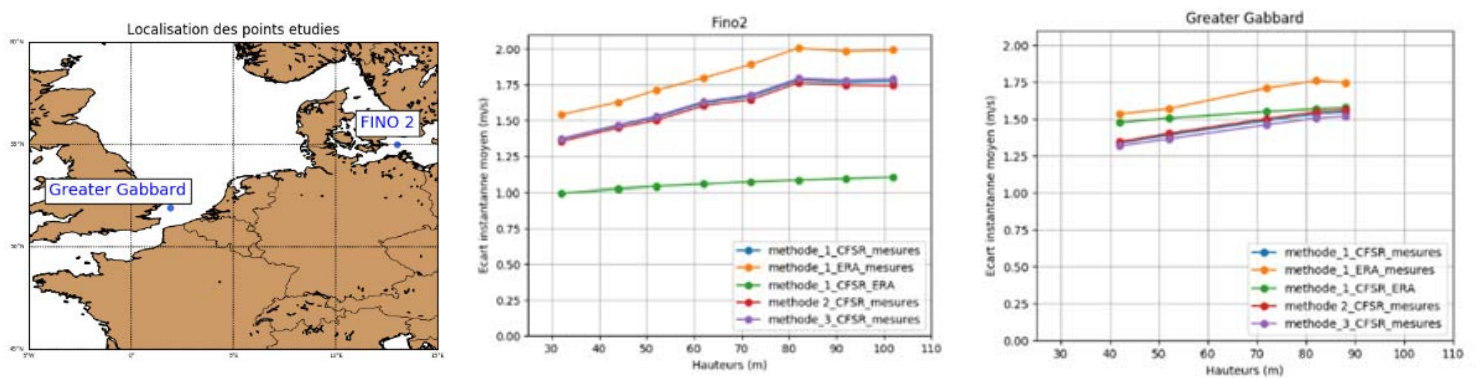

Figure 5. Localisation des points de mesures et variations des moyennes des valeurs absolues des écarts entre les vitesses de vent du modèle CFSR, du modèle Era Interim et des mesures in situ en fonction de l'altitude.

Les moyennes des valeurs absolues des écarts entre les vitesses augmentent avec l'altitude. Au point FINO 2 situé au large de l'Allemagne, les écarts sont plus faibles entre les ré analyses (courbe verte sur le graphique de gauche) qu'entre les ré analyses et les mesures. Au point de Greatter Gabbard, situé au large du Royaume-Uni, les écarts sont minimums entre les ré analyses du modèle CFSR et les mesures in situ (courbes bleue, rouge et violette sur le graphique de droite). L'utilisation des méthodes 1, 2 ou 3 ne modifie que faiblement ces écarts (courbes bleues, rouges et violette sur les deux graphiques).

Dans la suite des travaux les données utilisées sont les ré analyses du modèle CFSR.

\subsection{Facteur de charge théorique sur zone d'étude}

La production est évaluée pour chaque heure afin de déterminer le facteur de charge moyen de 1979 à 2010 (MATTAR C., BORVARAN D.). Les vitesses de vent prises en compte sont issues des ré analyses du modèle CFSR extrapolées verticalement par la méthode 1 en utilisant les équations (5) et (6) puis moyennées sur la surface du disque balayée par les pâles de l'éolienne.

Le facteur de charge estimé peut atteindre $60 \%$ de temps sur quelques secteurs de la mer du Nord. Cependant, il ne prend pas en compte :

- les variations du coefficient de puissance avec la vitesse de rotation des pâles ;

- l'extrapolation des vitesses et des directions du vent au-dessus de la couche limite atmosphérique ;

- la mise en arrêt des éoliennes liée à la maintenance des machines ;

- les pertes de production d'énergie liées aux effets de sillage au sein du parc, aux pertes électriques sur le réseau. 


\section{XVèmes Journées Nationales Génie Côtier - Génie Civil \\ La Rochelle, 29 au 31 mai 2018}

L'utilisation d'une courbe de puissance théorique aura tendance à surestimer le potentiel (JOURDIER, 2015). De plus, des données de vent plus précises pourraient permettre de mieux considérer des phénomènes de cisaillement, de turbulence ou des rafales qui peuvent impacter le fonctionnement des éoliennes.

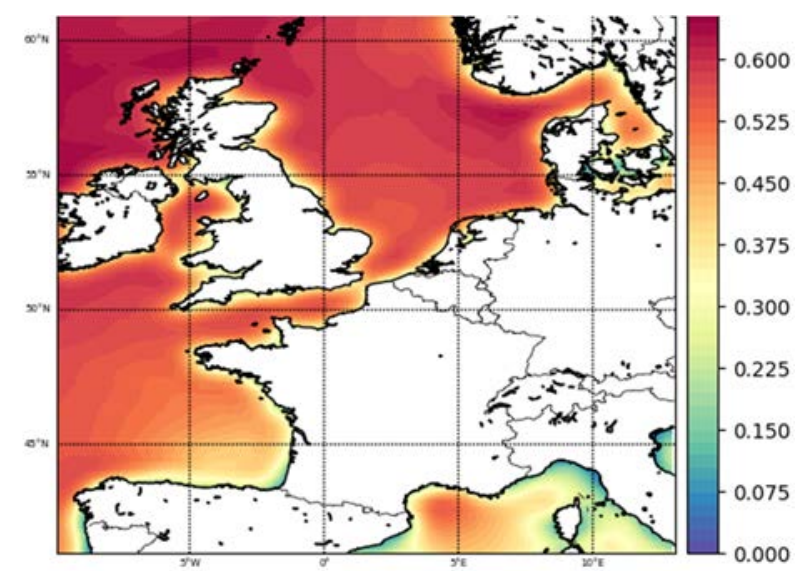

Figure 6. Représentation du facteur de charge théorique moyen de 1979 à 2010.

\subsection{Evolution du potentiel éolien}

Des travaux sur l'évolution du potentiel éolien ont été réalisés dans le cadre des projets CMIP (Coupled Model Intercomparison Project) de la communauté scientifique internationale. Ces travaux produisent des simulations climatiques à partir de différents modèles et servent à établir les conclusions des rapports du Groupe d'Experts Intergouvernemental sur l'évolution du Climat. Au cours des prochaines décennies une augmentation du potentiel en Europe du nord (en mer Baltique notamment) et une diminution dans le sud de l'Europe à proximité de la Méditerranée (HUEGING et al, 2013 ; CARVALHO et al, 2017) ont été identifiées. Par ailleurs, les variations saisonnières du vent devraient être plus marquées en mer Baltique et s'amoindrir dans le sud de l'Europe (CARVALHO et al, 2017).

\subsection{Site d'implantation des éoliennes}

Les données descriptives de la ressource en vent permettent de connaître les capacités de production des sites et donc les revenus potentiels d'un projet. Pour définir les meilleurs sites pour l'implantation des éoliennes, il faut également prendre en considération :

- les paramètres pouvant impacter sur les coûts des projets (conditions océaniques, nature des fonds et du substratum, distance à la côte pour le raccordement, ...),

- les activités, usages et enjeux environnementaux existants. 


\section{Thème 5 - Énergies et ressources marines}

En France, les zones propices pour le lancement des appels d'offres éoliens en mer sont issues d'un processus de concertation piloté par l'État et regroupant l'ensemble des parties prenantes.

\section{Conclusion}

Des ré analyses et des mesures de vent en mer sont disponibles en Europe afin d'étudier les variations spatiales et temporelles $d u$ vent et d'estimer un facteur de charge théorique pour les éoliennes en mer. La précision de ces données ne permet pas de réaliser une estimation fine et détaillée de la production à l'échelle des sites potentiels mais apporte des connaissances sur les fluctuations de la ressource en vent sur plusieurs dizaines d'années et pour différents pays. Les variations du vent, qui devraient évoluer avec le changement climatique, peuvent impacter fortement la production éolienne en mer en Europe et doivent donc être prises en considération dans le développement des projets.

\section{Références bibliographiques}

CARVALHO D., ROCHA A., GOMEZ-GESTEIRA M., SILVA SANTOS C. (2017), Potential impacts of climate change on European wind energy resource under the CMIP5 future climate projections. Renewable Energy, Vol. 101, pp 29-40. https://doi.org/10.1016/j.renene.2016.08.036

HENCKES P., KNAUT A., OBERMULLER.F, FRANCK C. (2018). The benefit of long-term high resolution wind data for electricity system analysis. Energy, Vol. 143, pp 934-942. https://doi.org/10.1016/j.energy.2017.10.049

HUEGING H., HASS R., BORN K., JACOB D., PINTO J. (2013), Regional changes in wind energy potential over Europe using regional climate model ensemble projections. Journal of applied meteorology and climatology, Vol. 52, pp 903-917. https://doi.org/10.1175/JAMC-D-12-086.1

JOURDIER B. (2015). Ressource éolienne en France métropolitaine: méthode d'évaluation du potentiel, variabilité et tendances. Thèse Ecole Doctoral Polytechnique, Paris. URL : http://www.theses.fr/2015EPXX0070

LAUGEL A. (2013). Climatologie des états de mer en Atlantique nord-est : analyse du climat actuel et des évolutions futures sous scénarios de changement climatique, par descente d'échelle dynamique et statistique. Thèse Université Paris Est, Paris. URL: http://www.theses.fr/2013PEST1120

MATTAR C., BORVARAN D. (2016). Offshore wind power simulation by using WRF in the central coast of Chile. Renewable Energy, Vol. 94, pp 22-31. https://doi.org/10.1016/j.renene.2016.03.005

MOLIN B. (2002). Hydrodynamique des structures offshore, Technip, Paris, $440 \mathrm{p}$. SUMNER J. (2006). Influence de la stabilité atmosphérique sur les pratiques du secteur éolien. Mémoire Ecole Technologique Supérieure, Quebec. http://espace.etsmtl.ca/id/eprint/484 